\title{
Relato de caso: alterações ósseas de sífilis congênita refratária ao tratamento em
}

\section{escolar de 7 anos}

\author{
Case report: bone alterations of congenital syphilis refractory to treatment in a 7-year-old \\ schoolboy
}

Reporte de caso: alteraciones óseas en sífilis congénita refractarias al tratamiento en un escolar de 7 años

\begin{abstract}
Resumo
Objetivos: Chamar a atenção para a importância do seguimento das crianças com sífilis congênita e apresentar um caso incomum de alterações ósseas que persistiram até os 7 anos de vida. Métodos: Estudo observacional, descritivo e retrospectivo utilizando revisão de prontuários de três pacientes com sífilis congênita e pesquisa bibliográfica, utilizando como bases de dados o Uptodate, PubMed, Medline, Lilacs, Scielo abrangendo o período de 2015 a 2021. Resultados: Descrevemos 3 irmãs com sífilis congênita que foram diagnosticadas e tratadas ao nascer e a primogênita, resgatada aos 7 anos, apresentava queixa de dor intensa ao movimentar os membros inferiores desde os 5 anos. Ao nascer teve o VDRL 1:4; pai VDRL não reagente (NR); mãe com VDRL 1:16 e inadequadamente tratada na gestação. A primogênita apresentou alterações metafisárias e VDRL no líquor negativo, sendo tratada com penicilina cristalina por 10 dias, entretanto, não cumpriu seguimento ambulatorial. Neste internamento, o exame físico sem alterações, VDRL NR e com radiografia dos ossos longos com imagem lítica em perna esquerda e direita sugestivas de sífilis. Foi indicada internação e realizado tratamento com penicilina cristalina $50.000 \mathrm{UI} / \mathrm{kg} / \mathrm{dose}$, de $6 / 6$ horas durante 10 dias. Aos 8 anos e 4 meses já não apresentava mais as alterações ósseas nem sintomas álgicos à deambulação. Na segunda gestação teve tratamento adequado e na terceira gestação o pai não foi tratado e a genitora recebeu apenas uma dose da medicação. Os dois conceptos contraíram sífilis congênita com alterações ósseas ao nascimento que nos seguimentos aos 24 meses e 6 meses de vida, respectivamente, já não foram mais detectadas. Conclusões: O seguimento das crianças com sífilis congênita deve ser realizado até o desaparecimento de todas as alterações clinicas, laboratoriais e radiológicas. Esta série de casos destaca a importância do diagnóstico e tratamento das gestantes e seu(s) parceiro(s) sexuais para eliminar a transmissão de mãe para filho como também do diagnóstico ao nascer dos casos de sífilis congênita e tratamento e seguimento adequado.
\end{abstract}

Palavras-chave: Sífilis congênita; Treponema pallidum; Osso e Ossos; Doenças sexualmente transmissíveis.

\begin{abstract}
Objectives: To draw attention to the importance of following up children with congenital syphilis and to present an unusual case of bone changes that persisted up to 7 years of life. Methods: Observational, descriptive and retrospective study using medical records review of three patients with congenital syphilis and literature search, using the Uptodate, PubMed, Medline, Lilacs, Scielo databases covering the period from 2015 to 2021. Results: We describe 3 sisters. with congenital syphilis that were diagnosed and treated at birth and the first-born, rescued at age 7 , complained of severe pain when moving her lower limbs since age 5 years. At birth he had VDRL 1:4; non-reactive VDRL parent (NR); mother with VDRL 1:16 and inadequately treated during pregnancy. The first-born had metaphyseal alterations and VDRL in negative CSF, being treated with crystalline penicillin for 10 days, however, she did not comply with
\end{abstract}


outpatient follow-up. During this hospitalization, the physical examination showed no alterations, VDRL NR and long bones radiography with lytic image in the left and right legs suggestive of syphilis. Hospitalization was indicated and treatment with crystalline penicillin 50,000 IU/kg/dose was performed, 6/6 hours for 10 days. At 8 years and 4 months, he no longer had bone changes or pain symptoms when walking. In the second pregnancy she had adequate treatment and in the third pregnancy the father was not treated and the mother received only one dose of medication. The two conceptuses contracted congenital syphilis with bone changes at birth which, in the follow-up at 24 months and 6 months of life, respectively, were no longer detected. Conclusions: The follow-up of children with congenital syphilis should be carried out until all clinical, laboratory and radiological alterations disappear. This case series highlights the importance of diagnosing and treating pregnant women and their sexual partner(s) to eliminate mother-to-child transmission as well as the diagnosis at birth of cases of congenital syphilis and adequate treatment and follow-up.

Keywords: Congenital syphilis; Treponema pallidum; Bone and bones; Sexually transmitted diseases.

\begin{abstract}
Resumen
Objetivos: Llamar la atención sobre la importancia del seguimiento de los niños con sífilis congénita y presentar un caso inusual de alteraciones óseas que persisten hasta los 7 años de vida. Métodos: Estudio observacional, descriptivo y retrospectivo mediante revisión de historias clínicas de tres pacientes con sífilis congénita y búsqueda bibliográfica, utilizando las bases de datos Uptodate, PubMed, Medline, Lilacs, Scielo cubriendo el período 2015 a 2021. Resultados: Describimos 3 hermanas. La sífilis congénita que fue diagnosticada y tratada al nacer y la primogénita, rescatada a los 7 años, se quejaba de fuertes dolores al mover sus miembros inferiores desde los 5 años. Al nacer tenía VDRL 1: 4; padre VDRL no reactivo (NR); madre con VDRL 1:16 y tratada inadecuadamente durante el embarazo. La primogénita presentó alteraciones metafisarias y VDRL en LCR negativo, siendo tratada con penicilina cristalina durante 10 días, sin embargo, no cumplió con seguimiento ambulatorio. Durante esta internación, el examen físico no mostró alteraciones, VDRL NR y radiografía de huesos largos con imagen lítica en pierna izquierda y derecha sugestiva de sífilis. Se indicó ingreso hospitalario y se realizó tratamiento con penicilina cristalina $50.000 \mathrm{UI} / \mathrm{kg} / \mathrm{dosis}$, 6/6 horas durante 10 días. A los 8 años y 4 meses ya no presentaba cambios óseos ni síntomas de dolor al caminar. En el segundo embarazo tuvo un tratamiento adecuado y en el tercer embarazo el padre no fue tratado y la madre recibió solo una dosis de medicación. Las dos concepciones contrajeron sífilis congénita con alteraciones óseas al nacer que, en el seguimiento a los 24 meses y 6 meses de vida, respectivamente, dejaron de detectarse. Conclusiones: El seguimiento de los niños con sífilis congénita debe realizarse hasta que desaparezcan todas las alteraciones clínicas, de laboratorio y radiológicas. Esta serie de casos destaca la importancia de diagnosticar y tratar a las embarazadas y su (s) pareja (s) sexual (es) para eliminar la transmisión maternoinfantil, así como el diagnóstico al nacimiento de los casos de sífilis congénita y el tratamiento y seguimiento adecuados.
\end{abstract}

Palabras clave: Sífilis congénita; Treponema pallidum; Huesos y huesos; Enfermedades sexualmente transmisibles.

\title{
1. Introdução
}

A sífilis, DST, que tem como pior desfecho a sífilis congênita (SC) pode ocorrer em 70 a $100 \%$ de crianças cujas mães foram inadequadamente tratadas ou não tratadas na gestação e se encontram na fase primária e secundária da doença. A alteração óssea é a anormalidade mais comum na sífilis congênita precoce não tratada, surgindo em 70\% a 100\% dos casos sintomáticos; tipicamente múltipla e simétrica, acometendo principalmente ossos longos. Pode ocorrer dor à movimentação ativa ou passiva dos membros e, por causa da dor, a criança pode apresentar-se irritada e tendente a imobilidade. A transmissão de mãe para filho (TMF) da sífilis durante a gravidez pode levar a resultados fetais graves no segundo ou terceiro trimestre, incluindo morte fetal precoce, natimorto, morte neonatal, parto prematuro, baixo peso ao nascer e infecção congênita em bebês sendo a segunda causa infecciosa mais comum de natimortos em todo o mundo (Gomez et al., 2013; Lawn et al, 2016).

No ano de 2016 ocorreram no mundo 355.000 aborto e feto morto, desses, 143.000 foram em gestantes com sífilis. O nascimento de crianças com sintomas de SC foi de 109.000, mortes neonatais, 14.000, prematuridade e baixo peso ao nascer, 41.000 (KORENROMP et. al, 2019). A SC é a segunda causa mais comum de mortes fetais evitáveis no mundo (CDC, 2018; LAWN, 2016). Estima-se que a incidência é de 6,3 milhões de casos por ano em todo o mundo. Nos últimos dez anos, a incidência da sífilis aumentou em mais de 150\% em alguns países desenvolvidos (Hoefer, 2021)

A Estratégia do Setor de Saúde Global da OMS, determinou como um dos quatro objetivos para 2030 a eliminação da SC. Considerada um grave problema de saúde pública colocou como meta em $80 \%$ dos países com alta taxa de incidência a 
redução para $\leq 0,5$ casos por 1.000 nascidos vivos (NV) (WHO, 2016). Nos Estados Unidos de 2013 a 2018 houve um aumento das taxas de SC de 261\% com uma taxa de incidência de 33 casos para 100.000 (KORENROMP et. al, 2019).

Segundo o Boletim epidemiológico no ano de 2019 foram notificados no SINAN 24.130 casos de SC no Brasil, taxa de mortalidade de 5,9 casos para 100.000 nascidos vivos (NV). A taxa de incidência de 8,2 e 14 casos para 1.000 NV no Brasil e Sergipe, respectivamente (Brasil, 2020).

Chama atenção a repetição de casos em uma mesma família. Para caracterizar a estrutura populacional global de T. pallidum, Beale et al., 2021 sequenciaram amostras de 23 países em todo o mundo e analisaram as sequências do genoma e concluíram que todos os 726 genomas pertenciam a apenas duas linhagens, Nichols e SS14, com o último dominando e ambos circulando globalmente (Aoroa, 2016; Beale, 2019; Beale et al,2021).

O objetivo deste relato é fornecer ferramentas para reconhecer a sífilis precoce e prevenir sua progressão para estágios avançados. Ainda no pré natal, as gestantes e parceiro(s) sexuais devem ser avaliados clinica e laboratorialmnte, com realização de exames para detecção da sífilis o mais precoce possivel, repetindo no terciero trimestre e no momento do parto, para evitar que a doença seja contraída pelo concepto.

\section{Metodologia}

Estudo descritivo, observacional e retrospectivo tipo relato de caso, conforme Yin, 2015 com coleta direta de dados. O artigo foi baseado na revisão de prontuários do acompanhamento hospitalar e ambulatorial de três crianças com diagnóstico de sífilis congênita baseado no protocolo do ministério da saúde vigente nos anos correspondentes aos nascimentos (2013, 2018, 2019). Também foi realizada pesquisa bibliográfica, utilizando como bases de dados o Uptodate, PubMed, Medline, Lilacs, Scielo abrangendo o período de 2015 a 2021 e utilizando os termos do vocabulário controlado do DeCS. Após autorização da direção clínica do Hospital onde encontra-se o ambulatório de especialidades para utilização dos prontuários e assinatura do Termo de Consentimento Livre e Esclarecido (TCLE) da genitora foi realizada a série de relato de casos.

\section{Apresentação do Caso 1}

Menina de 7 anos, com queixa principal de dor nas pernas à deambulação. Previamente tratada com penicilina cristalina ao nascer. Pai com Venereal Disease Research Laboratory (VDRL) NR no momento; mãe com VDRL 1:16 e inadequadamente tratada na gestação. Genitora com 23 e genitor com 26 anos quando tiveram a primeira filha. Ela fez tratamento para SC com penicilina cristalina por 10 dias, contudo, não cumpriu seguimento ambulatorial. Resgatada por este serviço com queixa dor intensa aos movimentos em membros inferiores há 2 anos. $\mathrm{O}$ exame físico mostrou criança ativa, bom estado geral, exame físico sem alterações. Foi observada na radiografia de ossos longos alterações radiológicas sugestivas de sífilis em membros superiores e perna esquerda e direita, caracterizadas por imagem lítica com osteoesclerose na região metafisária distal com reação periosteal difusa, que se estendia até epífise (Figura 1A). O exame sorológico, VDRL, foi negativo em líquor e sangue periférico. Internada para realizar tratamento com penicilina cristalina $50.000 \mathrm{UI} / \mathrm{kg} / \mathrm{dose}$, a cada 6 horas durante 10 dias e encaminhada para a seguimento no ambulatório do estudo. Aos 8 anos e 4 meses já não apresentava mais as alterações ósseas nem sintomas álgicos à deambulação (Figura 1B). 
Figura+1A:-Radiografias ' das extremida des superior esquerda' e direita' antes do retratamento -

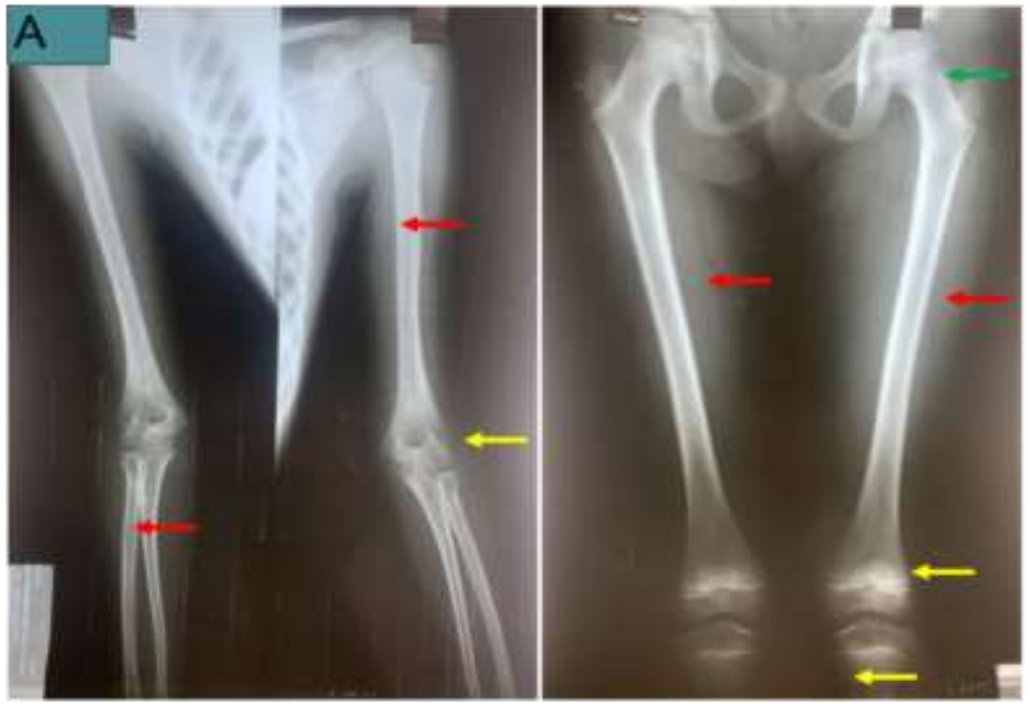

Legenda:- Nas" extremidades" superior" esquerda' e' direita" há periostite leve- difusa' dos' ossos' longos" (seta

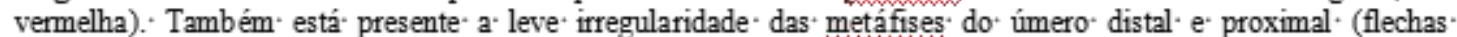

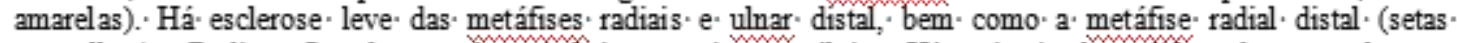
vermelhas). ' Radiografias ' dos membros' mferiores e cintura pélvica. " Há" periostite- leve difusa' dos' ossos longos (setas' vermelhas)." Também está presente a leve irregularidade - das metáfises femorais ' distal e proximal - (flechas verdes). Há esclerose-leve das mạțáfịșes tịbị ạis (setas amarelas)

Fonte: Autoria própria.

Figura+1B:- + Radiografias'das' extremidades'superiores 'e inferiores 'após retratamento

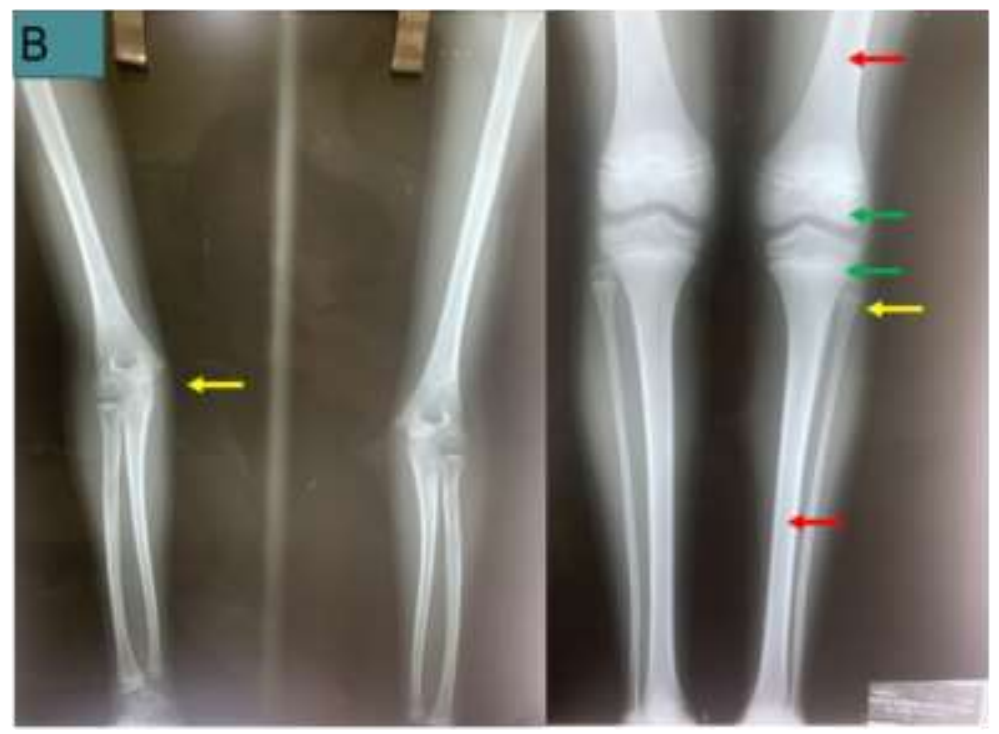

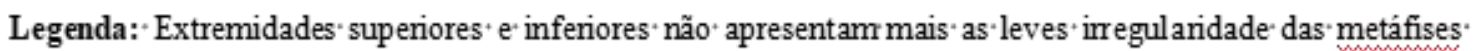
umerais $e^{-}$tibiais distal e proximal'(flechas verdes), bem como a metáfise umeral- proximal'(setas amarelas) + anteriormente' descritas. 'Nos 'membros 'inferiores 'após retratamento' compenicilina, 'há'remiss ão da' Reriostite' leve' difusa' dos" ossos longos'(setas"vermelhas).

Fonte: Autoria própria

\section{Apresentação do Caso 2}

A segunda das irmãs compareceu aos 2 anos, tendo perdido os primeiros meses de seguimento. Estava assintomática.

Nasceu de parto normal, a termo e com mãe em vigência de VDRL reagente, mas, mesmo tendo sido tratados ambos os pais na 
UBS, o pai tinha VDRL não reagente após tratamento e mãe com VDRL 1:16 e adequadamente tratada na gestação, com recitas que comprovaram o uso da medicação em dose e intervalos corretos.

Ao nascimento a segunda filha apresentava VDRL NR em líquor e sangue periférico, hemograma normal e alterações ósseas metafisárias em todos os ossos longos. Foi indicado internação e realizado tratamento com penicilina cristalina 50.000 $\mathrm{UI} / \mathrm{kg} /$ dose, a cada 12 horas até o sétimo dia de vida e a cada 8 horas a partir do oitavo dia de vida durante 10 dias. Realizado na alta o encaminhamento para seguir no ambulatório. Aos 2 anos de idade não apresentava alterações em ossos longos e o VDRL era não reagente.

\section{Apresentação do Caso 3}

Na gestação da filha caçula a mãe apresentou VDRL reagente 1:8. No acompanhamento pré-natal não foi realizado tratamento adequado, mesmo com história sexual de risco. Ao nascer a terceira filha realizou os exames preconizados (VDRL em sangue periférico e líquor, hemograma e radiografia de ossos longos). O VDRL teve a titulação de 1:2 e demais exames dentro da normalidade. Ao nascimento recebeu tratamento com penicilina cristalina por 10 dias, Aos 6 meses apresentava VDRL NR. O seguimento ficou prejudicado devido a pandemia vigente da COVID-19 (Tabela 1).

Tabela 1: Características clínicas e radiológicas dos casos estudados.

\begin{tabular}{|c|c|c|c|}
\hline & Caso 1 & Caso 2 & Caso 3 \\
\hline Pré-natal & SIM & SIM & SIM \\
\hline $\begin{array}{l}\text { Tratamento materno na } \\
\text { Gestação }\end{array}$ & Adequado & Inadequado & Não tratou \\
\hline Tratamento paterno & SIM & SIM & NÃO \\
\hline VDRL da mãe no parto & $01: 16$ & 01:08 & 01:04 \\
\hline VDRL do RN ao nascimento & 01:04 & NR & 01:02 \\
\hline VDRL (idade) & $7 \operatorname{anos} \mathrm{NR}$ & 2anos NR & 1mês NR \\
\hline Lesão Óssea & SIM & SIM & SIM \\
\hline Neurosífilis & NÃO & NÃO & NÃO \\
\hline Alteração Radiológica & $\begin{array}{l}\text { Osteoesclerose metafisária e } \\
\text { epifisária difusa }\end{array}$ & $\begin{array}{l}\text { Osteoesclerose } \\
\text { metafisária difusa }\end{array}$ & Metáfises em taça \\
\hline Osso(s) Afetado(s) & $\begin{array}{l}\text { Antebraço, braço, úmero, } \\
\text { fêmur, coxa e pernas direitos } \\
\text { e esquerdos }\end{array}$ & $\begin{array}{l}\text { Antebraço, } \quad \text { braço, } \\
\text { fêmur, coxa e pernas } \\
\text { direitos e esquerdos }\end{array}$ & $\begin{array}{l}\text { Fêmur, coxa e pernas } \\
\text { direitos e esquerdos }\end{array}$ \\
\hline
\end{tabular}

Fonte: Autoria própria.

\section{Discussão}

A sífilis congênita é marcadora de desenvolvimento de um país pois o tratamento é realizado com penicilina considerada uma medicação básica da saúde e exames laboratoriais de fácil acesso. Quando a gestante é adequadamente tratada na gravidez a SC pode ocorrer em apenas 1 a $2 \%$ dos casos. Silveira el al., 2021, em estudo com dados secundários do SINAN concluíram que $72,7 \%$ das gestantes sergipanas com diagnóstico de VDRL reagente tiveram diagnóstico tardio mesmo tendo realizado pré-natal. Segundo o Protocolo do Ministério da Saúde a gestante adequadamente tratada seria a que fez uso de 
penicilina benzatina até 30 dias antes do parto, com intervalo e dose correta, avaliação quanto ao risco de reinfecções e documentação comprovando a queda da titulação do VDRL materno em duas diluições até o sexto mês e quatro diluições até doze meses após a conclusão do tratamento.

Dos três casos relatados, duas das irmãs apresentaram manifestações precoces e uma manifestação tardia da sífilis congênita. Entre elas apenas uma era sintomática. Todas nasceram de parto normal, a termo e com mãe em vigência de VDRL reagente. Mesmo assim, em nenhum dos três casos foi realizado acompanhamento pré-natal adequado, mesmo com história sexual de risco. Em dois casos não foram solicitados exames nas consultas de pré-natal em que a mãe compareceu à UBS. Já quanto ao seguimento das crianças, o mesmo só ocorreu nos primeiros dois anos de vida e logo em seguida não compareciam às consultas.

Faz-se necessário incluir o pai nas consultas de pré-natal e realizar tratamento adequado como também avaliar as manifestações de sintomas e falha de tratamento. O VDRL deve ser realizado mensalmente nas gestantes que foram diagnosticadas e tratadas para sífilis para acompanhar a queda da titulação. As gestantes apresentam em 2 a $14 \%$ falhas terapêuticas que necessitam ser diagnosticadas e retratadas prontamente para evitar a SC. É considerado que o tratamento falhou se o VDRL aumentar duas diluições no seguimento (Ghanem, et al 2020 \& Diorio et al, 2018).

A edição 2020 da diretriz europeia sobre a gestão da sífilis é uma atualização da edição de 2014 e inclui dentre as principais modificações e atualizações a importância da implementação de pontos de atendimento onde possam ser realizados duplo testes, treponêmico e não treponêmico. Frisa também a escassez do tratamento regular com penicilina G benzatina (BPG) em alguns países europeus e a exclusão da azitromicina como um tratamento alternativo em qualquer estágio da sífilis e reitera a profilaxia pré-exposição ou pós-exposição imediata com doxiciclina em populações com alto risco de adquirir sífilis (Janier et al., 2020)

No Brasil, a realidade não é diferente, garantir o seguimento de todas as crianças expostas à SC, excluída ou confirmada é extremamente relevante para eliminação dessa doença. A detecção dos sinais e sintomas mais tardios, independentemente da primeira avaliação e/ ou tratamento na maternidade é necessário para prevenir as comorbidades da SC. A testagem simultânea da mãe e da criança, no pós-parto imediato, além de um exame físico minucioso, configura o melhor cenário para evitar as alterações ósseas, auditivas, visuais e de crescimento e desenvolvimento que podem surgir nas crianças acometidas por essa doença (Andrade et al 2018. \& Ministério da Saúde (BR) 2016. \& Diorioet al, 2018).

\section{Considerações Finais}

Identificar corretamente a situação é decisivo para a conduta visto que os casos de sífilis congênita, precoce ou tardia, a conduta deverá ser a internação e tratamento com penicilina cristalina por 10 dias.

No teste não treponêmico, um título maior que o materno em pelo menos duas diluições (ex.: materno 1:4, RN maior ou igual a 1:16) é indicativo da doença mesmo em mães adequadamente tratadas. A testagem simultânea da mãe e da criança, no pós-parto imediato, com o mesmo tipo de teste não treponêmico é o que melhor determina os achados sorológicos da criança. Mesmo a criança sendo corretamente tratada ao nascer ela deve ser seguida para avaliar falha terapêutica. A primogênita da série de caso apresentava alterações ósseas e dor em membros inferiores aos 7 anos, quando foi retratada. Um ano após o segundo tratamento ele não apresentava mais sintomas álgicos e a radiografia dos ossos longos era normal.

Tendo em vista que crianças expostas à sífilis podem desenvolver sinais e sintomas mais tardios, é essencial garantir o seguimento ambulatorial dos referidos pacientes, independentemente da doença ser excluída ou confirmada em uma avaliação inicial ainda na maternidade. 
Esta série de relatos de caso destaca a importância do rastreamento em mulheres grávidas para eliminar a transmissão de mãe para filho, e alcançar a tão desejada meta de 0,5 a menos casos de SC para $1000 \mathrm{NV}$, a qual é considerada a taxa para que um país atinja a eliminação da doença.

A infecção óssea, particularmente dos ossos longos das pernas e braços, apesar de ser uma característica comum da sífilis em estágio terciário e da sífilis congênita no passado, raramente é encontrada clinicamente em idades mais avançadas devido à terapia antibiótica eficaz. Relatamos o caso de uma criança de 7 anos que apresentou dor aguda há um ano na perna esquerda. O teste de aglutinação das partículas do Treponema pallidum foi positivo e a radiografia mostrou uma osteólise patológica da tibiofíbula esquerda e direita. Os sintomas da paciente desapareceram completamente após um curso de penicilina e 10 dias. Sugerimos que lesões ósseas, podem ser a única manifestação da sífilis latente tardia. Assim, em trabalhos futuros faz-se necessária uma avaliação mais ampla do seguimento bem como um novo olhar para fatores determinantes da doença. Os dados genômicos do Treponema pallidum, o agente causador da sífilis, são limitados e cobrem principalmente amostras da Europa e da América do Norte e deste século. A avaliação gênica destas séries de casos familiares seria imperativo.

\section{Referências}

Andrade, A. L. M. B., Magalhães, P. V. V. S., Moraes, M. M., Tresoldi, A. T. \& Pereira, R. M. (2018). Diagnóstico tardio de sífilis congênita: uma realidade na atenção à saúde da mulher e da criança no Brasil. Rev Paul Pediatria. 36(3):376-81.

Arora, N. et al. Origin of modern syphilis and emergence of a pandemic Treponema pallidum cluster. (2016). Nat. Microbiol. (2), 16245

Beale, M. A. et al. Genomic epidemiology of syphilis reveals independent emergence of macrolide resistance across multiple circulating lineages (2019). Nat. Commun. (10), 3255.

Beale, M.A., Marks, M., Cole, M. J. et al. (2021). Global phylogeny of Treponema pallidum lineages reveals recent expansion and spread of contemporary syphilis. Nat Microbiol (6), 1549-1560. https://doi.org/10.1038/s41564-021-01000-z

Centers for Disease Control and Prevention (2018). National Notifiable Diseases Surveillance System (NNDSS). Syphilis 2018 Case Definition. https://wwwn.cdc.gov/nndss/conditions/syphilis/case-definition/2018/2018.

Diorio, D., Kroeger, K., \& Ross, A. (2018). Social Vulnerability in Congenital Syphilis Case Mothers: Qualitative Assessment of Cases in Indiana, 2014 to 2016. Sex Transm Dis. 2018 Jul;45(7):447-451.

Eickoff, C. A., \& Decker, C. F. (2010). Syphilis. Dis Mon. 2016, (62):280-6.

Forrestel, A. K., Kovarik, C. L., \& Katz, K. A. (2020). Sexually acquired syphilis: historical aspects, microbiology, epidemiology, and clinical manifestations. $J$ Am Acad Dermatol. 2020, (82):1-14.

Ghanem, K. G., Ram, S., \& Rice, P. A. (2020). The modern epidemic of syphilis. N Engl J Med. 2020, (382):845-54.

Gomez, G. B., Kamb, M. L., Newman, L. M., Mark, J., Broutet, N., \& Hawkes, S. J. Untreated maternal syphilis and adverse outcomes of pregnancy: a systematic review and meta-analysis (2013). Bull World Health Organ. 91(3):217-26. 10.2471/BLT.12.107623

Hofer, U. Bottleneck and spread of Treponema pallidum. (2021). Nat Rev Microbiol. https://doi.org/10.1038/s41579-021-00673-x

Janier, M., Unemo, M., Dupin, N., Tiplica, G., Potočnik, M. \& Patel, R. (2021), 2020 European guideline on the management of syphilis. J Eur Acad Dermatol Venereol, 35: 574-588. https://doi.org/10.1111/jdv.16946

Kimball, A., Torrone, E., Miele, K., et al. (2020). Missed Opportunities for Prevention of Congenital Syphilis - United States, 2018. MMWR Morb Mortal Wkly Rep. (69):661.

Korenromp, E. L., Rowley, J., Alonso, M., Mello, M. B., Wijesooriya, N. S., Mahiané, S. G., Ishikawa, N., Le, L. V., Newman-Owiredu, M., Nagelkerke, N., Newman, L., Kamb, M., Broutet, N., \& Taylor, M. M. (2019). Global burden of maternal and congenital syphilis and associated adverse birth outcomesEstimates for 2016 and progress since 2012. PloS one, 14(2), e0211720. https://doi.org/10.1371/journal.pone.0211720

Lawn JE, Blencowe H, Waiswa P, Amouzou A, Mathers C, Hogan D, et al. Stillbirths: rates, risk factors, and acceleration towards 2030 (2016). Lancet. 387(10018):587-603. 10.1016/S0140-6736(15)00837-5.

Ministério da Saúde (BR) (2016). Secretaria de Vigilância em Saúde. Departamento de Vigilância, Prevenção e Controle das Doenças Sexualmente Transmissíveis, Aids e Hepatites Virais. Manual técnico para diagnóstico da sífilis. Brasília (DF): Ministério da Saúde; 2016.

Peeling, R.W., Mabey, D., Kamb, M.L., Xiang-Sheng, C., Radolf, J. D., \& Benzaken, A.S.(2017). Nature Reviews Disease. 2017; (3)

Silveira, K. B. da, Silva, J. R. S., Reis, F. P., Melo, Ítalo F. L. de, Santos, T. C. de M., Souza, M. de J., \& Feitosa, V. L. C. (2021). Epidemiologia da Sífilis Congênita no estado de Sergipe. Research, Society and Development, 10(14), e562101422061. https://doi.org/10.33448/rsd-v10i14.22061 
Research, Society and Development, v. 10, n. 17, e253101724851, 2021 (CC BY 4.0) | ISSN 2525-3409 | DOI: http://dx.doi.org/10.33448/rsd-v10i17.24851

Watts, P. J., Greenberg, H. L., \& Khachemoune, A. (2016). Unusual primary syphilis: presentation of a likely case with a review of the stages of acquired syphilis, its differential diagnoses, management, and current recommendations. Int J Dermatol. 2016, (55):714-28.

WHO. (2016). Guidelines for the treatment of Treponema pallidum (syphilis). http://apps.who.int/iris/bitstream/10665/249572/1/9789241549806eng.pdf?ua=1.

Yin, R. K. (2015). O estudo de caso. Bookman. 